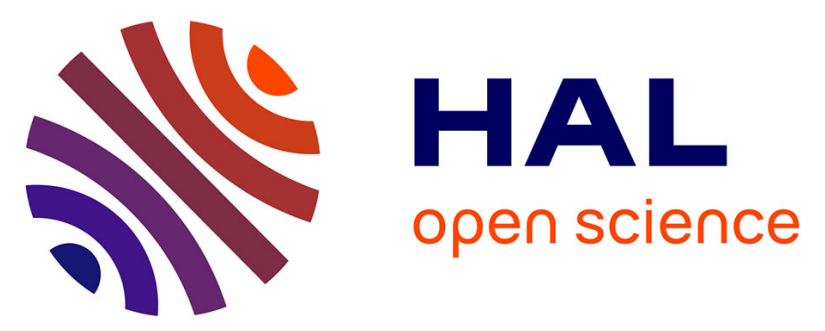

\title{
A new rotary tribometer to study the wear of reinforced rubber materials
}

Zélie Mané, Jean-Luc Loubet, Chrystelle Guerret, Laurent Guy, Olivier Sanseau, Ludovic Odoni, Loïc Vanel, Didier Long, Paul Sotta

\section{- To cite this version:}

Zélie Mané, Jean-Luc Loubet, Chrystelle Guerret, Laurent Guy, Olivier Sanseau, et al.. A new rotary tribometer to study the wear of reinforced rubber materials. Wear, 2013, 306 (1-2), pp.149-160. 10.1016/j.wear.2013.07.012 . hal-02389870

\section{HAL Id: hal-02389870 https://hal.science/hal-02389870}

Submitted on 9 Nov 2020

HAL is a multi-disciplinary open access archive for the deposit and dissemination of scientific research documents, whether they are published or not. The documents may come from teaching and research institutions in France or abroad, or from public or private research centers.
L'archive ouverte pluridisciplinaire HAL, est destinée au dépôt et à la diffusion de documents scientifiques de niveau recherche, publiés ou non, émanant des établissements d'enseignement et de recherche français ou étrangers, des laboratoires publics ou privés.

\section{(c)(1)}

Distributed under a Creative Commons Attribution| 4.0 International License 


\title{
A new rotary tribometer to study the wear of reinforced rubber materials
}

\author{
Z. Mané ${ }^{\mathrm{a}, \mathrm{b}}$, J.-L. Loubet $^{\mathrm{a}}$, C. Guerret ${ }^{\mathrm{a}}$, L. Guy ${ }^{\mathrm{d}}$, O. Sanseau ${ }^{\mathrm{b}}$, L. Odoni ${ }^{\mathrm{b}}$, L. Vanel ${ }^{\mathrm{b}, \mathrm{c}}$, \\ D.R. Long ${ }^{\mathrm{b}}$, P. Sotta ${ }^{\mathrm{b}, *}$ \\ a Laboratoire de Tribologie et Dynamique des Systèmes (LTDS) UMR 5513, CNRS/École Centrale de Lyon, 36, avenue Guy de Collonge, 69134 Écully, France \\ b Laboratoire des Polymères et Matériaux Avancés (LPMA), CNRS/Rhodia-Solvay UMR 5268, RÉI Centre Lyon, 85 avenue des frères Perret, 69192 Saint Fons Cedex, \\ France \\ ' Institut Lumière Matière, UMR5306 Université Lyon 1-CNRS, Université de Lyon, 69622 Villeurbanne Cedex, France \\ d Solvay Silica, BP 52, 15 Rue Pierre Pays, 69660 Collonges au Mont d'Or, France
}

A rotary tribometer has been developed in order to reproduce abrasion wear at the interface between the reinforced rubber material of a tire tread and the road surface, under controlled environmental parameters. The characteristics of the device are described in this paper. It consists in a spherical indenter sliding on the rubber material under study. The control parameters are the normal load, the ratio between the indenter radius and the sample thickness, the sliding velocity, the number of passages, the time interval between two passages, and the temperature. The friction coefficient and weight loss are measured all along the wear test. Wear patterns are observed in situ with an optical pen. We show preliminary results on reinforced natural rubber materials which illustrate the potentialities of the setup. Different wear patterns could be created and observed, according to the conditions.

\section{Introduction}

Efforts transmitted by a vehicle are located in the contact zone between the tire tread surface and the road, which is generally of the order of a few hundred squared centimeters. The tread must have high resistance to fatigue, abrasion wear, tearing and impact and should also withstand quite high temperatures [1-5]. Also, the friction coefficient between the tread and the road must be high in order to provide grip and cornering stability. Wear occurs in this contact zone. When expressed in terms of tire thickness loss per revolution, wear rates observed in soft usage conditions are of the order of a few $10^{-10} \mathrm{~m}$ per cycle, which is of the order of an atomic dimension, while elementary components of the material (polymer chains, filler aggregates) have sizes from a few nanometers to a few hundreds of nanometers. Also, the size of debris pieces is of the order of micrometers to tens of micrometers typically. It follows that wear is not a continuous process, but is an average result of discontinuous phenomena, at intermediate (submillimetric) scales.

\footnotetext{
* Corresponding author. Tel.: +33 472896466; fax: +33 472896963.

E-mail address: Paul.SOTTA-EXTERIEUR@eu.rhodia.com (P. Sotta).
}

Reinforced elastomers exhibit very specific wear patterns [6]. Wear patterns and abrasion weight loss depend on three sets of parameters [4]: (1) the constitutive equation of the material, which itself depends on material formulation [7-9]; (2) the mechanical loading (normal pressure, sliding velocity, slipped length [10-12], contact surface [13-16]); and (3) the environmental conditions (temperature, hygrometry, atmospheric composition) [17]. The various wear patterns observed in reinforced rubbers may be classified into four characteristic patterns [1-3]: (1) Grooves are patterns parallel to the sliding direction, associated to extremely severe wear conditions on rough surfaces, with dimensions from micrometers to millimeters [18]; (2) Ridges form regularly spaced arrays of asymmetric steps perpendicular to the sliding direction, with the steeper side (riser) facing the oncoming sliding indenter, as shown in Fig. 1 [17,19-22]. The distance between ridges ranges from micrometers to millimeters and their height is typically ten times smaller than this distance. Ridges are created in less severe conditions than for grooves; (3) Craters are roughly circular patterns, with diameters ranging from tens to hundreds of micrometers and a depth of about a tenth of the diameter, associated to very soft wear conditions and small sliding velocities [1]; and (4) Rollers are formed by wear fragments sticking on the rubber surface [23,24].

The few studies on the mechanisms of creation of these wear patterns have been generally focused on ridges [25,2,17,19-22]. One study has been devoted to crater formation [1]. 

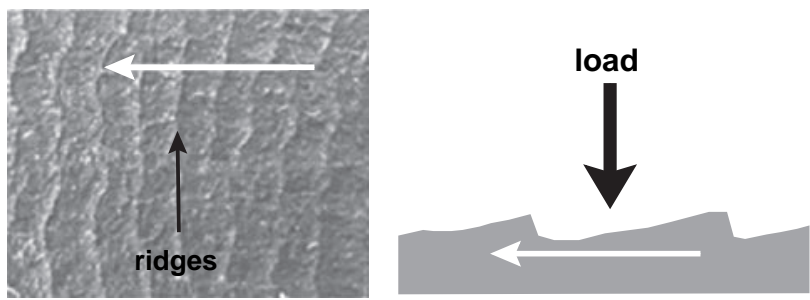

Fig. 1. Left: A representative example of wear ridges seen from above the surface; right: schematics of the lateral view. The direction of the sliding indenter is indicated by horizontal arrows.

In order to model the tread/road contact, several issues, related to the complexities of both the materials and the mechanical problem at the tread/road interface, have to be addressed.

Wear has been generally studied in laboratory conditions using a sliding indenter rubbing on the rubber surface. Contact sliding is a necessary condition to observe wear, i.e. abrasion weight loss. Indeed, it has been shown that wear is univoquely associated to slip and does not occur in indentation experiments in the absence of slip $[1,3]$. In the tread/road contact zone, sliding may be global, due to wet skid for example. However, it is essential to realize that, even in the absence of skid and of tangential efforts transmitted by the vehicle, some local slippage occurs in the contact zone [26].

As regards materials, reinforced elastomers used in tire treads are cross-linked (vulcanized) elastomers reinforced by solid particles or aggregates (fillers). They show remarkable, complex mechanical properties [27-30]. First, reinforcement (as quantified by the ratio of the elastic modulus of the material to that of the pure, unfilled matrix) may be very large (up to a factor 100) in a limited temperature range just above $T_{g}$ and it extends over a broad temperature range, with smaller values however. In practical applications, reinforced elastomers are often used in this broad temperature range. Then, the elastic modulus drops quite strongly at a few percents strain amplitude (Payne effect) [31]. These materials can sustain elastic deformations up to a few 100 percent, with a non-linear constitutive law and large dissipation. After large amplitude strain, they show partially recoverable softening (Mullins effect), as well as a significant residual strain (plasticity or residual set) [32,33] and long relaxation times $[34,35]$. Finally, they exhibit an outstanding resistance to tear and wear. All these features are of primary importance as regards rolling resistance, grip and durability [30].

To obtain high reinforcement, reinforcing fillers must be typically $100 \mathrm{~nm}$ in size, and strong enough filler/matrix interactions must be promoted [33]. Carbon black (CB) aggregates have predominantly been used $[33,36]$. Using silica may provide an enhanced compromise of usage properties [37], essentially because it offers more flexibility to finely tune the dissipation in various temperature and/or frequency domains.

Several models have been proposed to rationalize this remarkable mechanical behavior. It has been proposed recently that all these effects may be explained by the presence of a glassy (immobilized) layer of elastomer matrix around filler particles, which can build glassy bridges between particles on nanometric distances and are plasticized under local stress [38,39].

The objective of this paper is to present the characteristics of a new experimental device (tribometer), which has been developed in order to mimic the interface between the reinforced rubber material of a tire tread and the road surface (in soft driving conditions and in straight line), under controlled environmental parameters. It is a sphere-on-plate device operating on a thin layer sample, which allows applying a relatively high contact pressure and a long slip length. The involved contact mechanics is discussed in details. Preliminary results obtained on some representative materials are shown. We claim that this device has a number of characteristic features which make it suitable to generate and characterize wear patterns representative of real usage conditions and to allow proper identification and characterization of these wear patterns.

The paper is organized as follows. The studied materials are described in Section 2.1, their mechanical properties are described in Section 2.2. The tribometer is described in Section 3.1. The working conditions are discussed in details (Section 3.3). Results of representative tribological tests realized in reinforced natural rubber samples are presented in Section 4.

\section{Materials}

\subsection{Formulations}

The studied materials are Natural Rubber (NR, grade SMR 5L) vulcanized with a conventional sulfur/accelerator process and with two types of reinforcing systems: carbon black N234 (CB sample); precipitated silica (Z1165MP from Rhodia, specific surface $160 \mathrm{~m}^{2} / \mathrm{g}$ ) treated with triethoxysilylpropyltetrasulfur (TESPT), which covalently links the silica surface to the NR matrix. Two different volume fractions of silica have been used (SIL-1 and SIL-2 samples). The materials are mixed in an Internal Mixer (Banburry 11 ) and vulcanized in the form of $2 \mathrm{~mm}$ thick sheets. We know that homogeneous cross-link densities are obtained in the processing conditions which are used $[40,41]$. Formulations are described in Table 1. Note that mechanical data obtained in a non-reinforced (unfilled) Natural Rubber sample vulcanized in similar conditions (1.5 phr Sulfur, 2 phr CBS) are also shown for comparison in Section 2.2.

\subsection{Mechanical characterization}

The mechanical behavior of the materials is important to understand the wear behavior and has been extensively characterized in the linear and non-linear regimes. The CB and SIL-1 samples have been formulated in order to have the same Shore hardness, while material SIL-2 is slightly harder (see Table 1). These formulations have been chosen in order to illustrate the effect of changing the type of filler (carbon black vs. precipitated silica)

Table 1

Formulations of the studied materials CB, SIL-1 and SIL-2. Units are in phr (grams per hundred grams of rubber).

\begin{tabular}{llcrr}
\hline & Ingredients & CB & SIL-1 & SIL-2 \\
\hline \multirow{2}{*}{ Matrix } & NR (SMR 5L) & 100 & 100 & 100 \\
Filler & Silica Z1165 MP & & 50 & 55 \\
& Carbon black N234 & 50 & & \\
Additives & TESPT & & 4.0 & 4.4 \\
& 6PPD & 1.9 & 1.9 & 1.9 \\
& Stearic acid & 4.0 & 4.0 & 4.0 \\
& ZnO & 4.0 & 4.0 & 4.0 \\
& Sulfur & 1.5 & 1.5 & 1.5 \\
& CBS & 1.5 & 2.0 & 2.2 \\
& TBzTD & & \\
& Filler volume fraction & 0.2 & 0.2 & 0.2 \\
& T $_{\text {( }}{ }^{\circ}$ C) & -47 & 0.20 & 0.22 \\
& Hardness (Shore A) & 70 & -47 & -47 \\
& & & 70 & 72
\end{tabular}

a $N$-(1,3-dimethylbutyl)- $N^{\prime}$-phenyl-P-phenylenediamine (antioxidant).

${ }^{b} \mathrm{~N}$-cyclohexyl-2benzothiazyl (primary accelerator). Amount is tuned to compensate for adsorption by silica.

c Tetrabenzylthiuram disulfide (secondary accelerator). Some characteristic data are shown also (three bottom lines). 
or varying the volume fraction of silica (materials SIL-1 vs. SIL-2) on the tribological behavior of the materials.

Storage $\left(E^{\prime}\right)$ and loss $\left(E^{\prime \prime}\right)$ oscillatory tensile moduli at amplitude $0.1 \%$ and frequency $10 \mathrm{~Hz}$ are plotted as a function of temperature (from $-80^{\circ} \mathrm{C}$ to $80^{\circ} \mathrm{C}$ ) in Fig. 2 for the three studied materials. The three materials have the same glass transition temperature $T_{g}=-47{ }^{\circ} \mathrm{C}$ as defined by the maximum of the loss factor $\tan \delta=E^{\prime \prime} / E^{\prime}$. Altogether, all reinforced materials have a very similar behavior. The modulus of a non-reinforced (unfilled) Natural Rubber elastomer vulcanized under similar conditions is shown for comparison. The reinforcement (ratio of the reinforced modulus to that of the unfilled system) is of order 10-20 typically, in the temperature range of interest. Silica-filled samples (SIL-1 and SIL-2) have a higher dissipation (i.e. a higher maximum in $\tan \delta$ ) than the $\mathrm{CB}$ sample in the vicinity of $T_{g}$, while the CB sample becomes more dissipative at high temperature. Tensile stress-strain curves up to failure at room temperature are shown in Fig. 3 for the three studied materials. In all three materials, the stress at break is close to 28-29 MPa. Both silica samples, however, have lower moduli (less reinforcing constitutive laws) at high

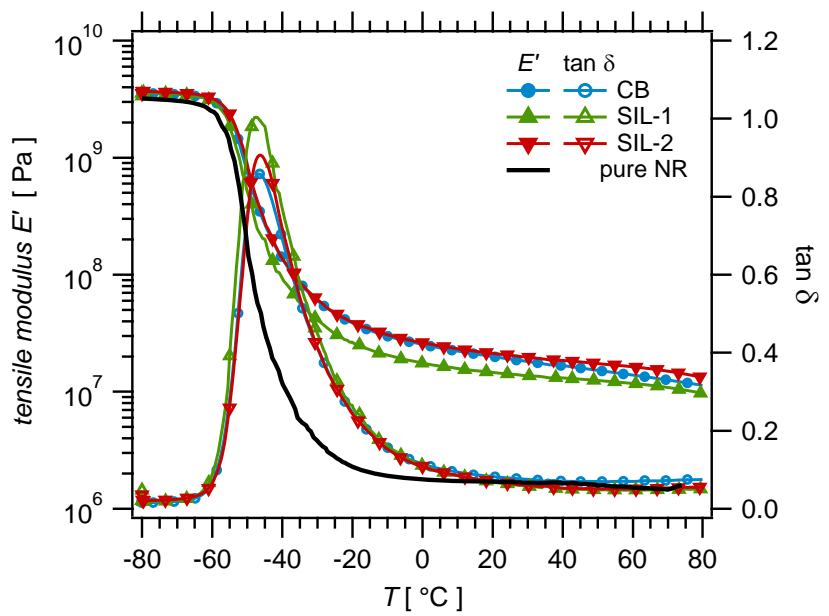

Fig. 2. Storage tensile moduli $E^{\prime}$ and loss factor $\tan \delta$ as a function of temperature (from $-80{ }^{\circ} \mathrm{C}$ to $80{ }^{\circ} \mathrm{C}$ ) in the linear regime (amplitude $0.1 \%$, frequency $10 \mathrm{~Hz}$ ), for the 3 studied materials CB, SIL- 1 and SIL-2. The modulus of a similar unfilled NR elastomer is shown for comparison.

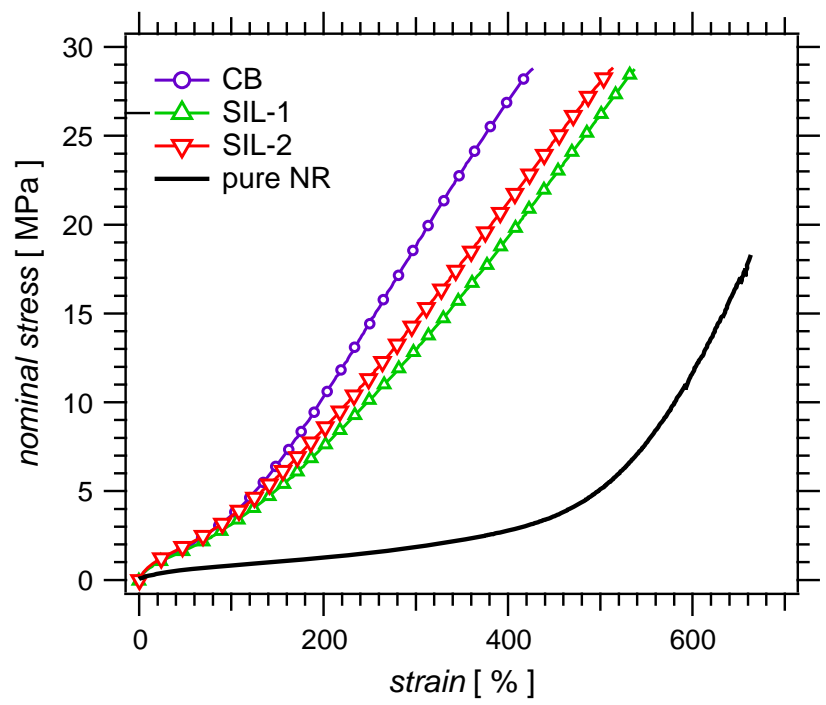

Fig. 3. Nominal stress as a function of the strain up to tensile failure at room temperature for the 3 studied materials CB, SIL- 1 and SIL-2. The curve of a similar unfilled NR elastomer is shown for comparison. strain, which results in larger strains at break in these materials as compared to the CB sample. Altogether, all materials have comparable, good performances, due to good cross-linking homogeneity and filler dispersion state. The tensile stress-strain curve of a nonreinforced (unfilled) Natural Rubber elastomer vulcanized under similar conditions is shown for comparison.

The Payne effect (drop of the modulus when an oscillatory strain of increasing amplitude is applied) has been characterized in the three materials. As we shall see below, in tribological tests, the typical applied strain values are those in which the Payne effect is observed. Storage $\left(G^{\prime}\right)$ and loss shear moduli $\left(G^{\prime \prime}\right)$ are plotted as a function of the amplitude of the oscillatory shear strain in Fig. 4, at frequency $10 \mathrm{~Hz}$ and at room temperature. Note that $E^{\prime}=3 G^{\prime}$ since the material is quasi-incompressible (Poisson coefficient $\nu \sim 0.5$ ). Both SIL-1 and SIL-2 samples have a smaller Payne effect (a smaller relative drop of $G^{\prime}$, together with a significantly less pronounced maximum in $G^{\prime \prime}$ ) than the CB sample. Note that the formulations of the samples have been adapted to match the storage moduli in the $10 \%$ deformation range, rather than in the linear regime. It follows that silica-filled samples have lower moduli than the CB-filled sample at very small amplitudes (in the linear regime).

The variation of the shear modulus $G^{\prime}$ as a function of the strain amplitude (Fig. 4) can be fitted with a heuristic function of the form [42]:

$G^{\prime}=\frac{P_{0}}{1+P_{1} \epsilon^{P_{3}}}+P_{2}$

Fits of the $G^{\prime}$ vs. strain amplitude curves are shown in Fig. 4. The obtained fitting coefficients for the three samples are summarized in Table 2. These data will be used when analyzing indentation curves in Section 3.3.

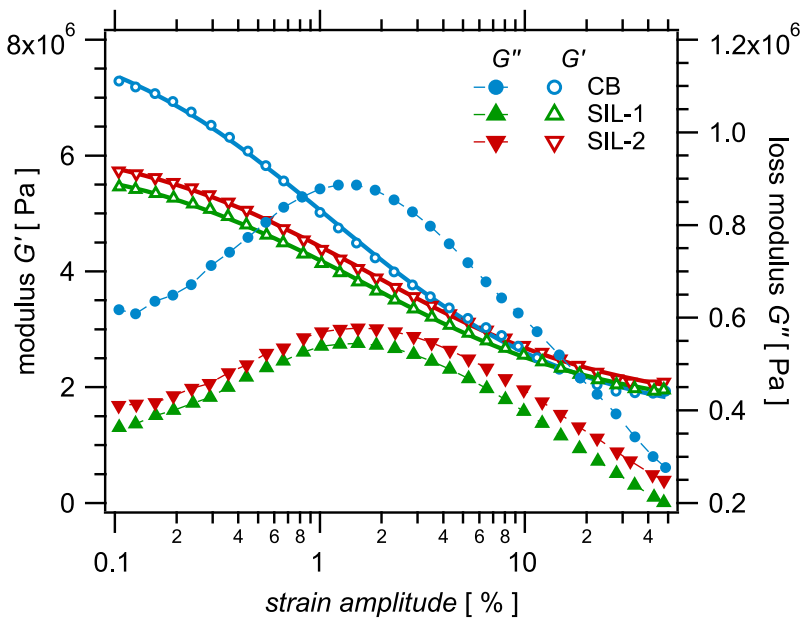

Fig. 4. Storage ( $G^{\prime}$, full curves) and loss ( $G^{\prime \prime}$, dashed curves) shear moduli as a function of the strain amplitude (Payne effect) at room temperature for the 3 studied materials CB, SIL-1 and SIL-2. Curves through symbols for $G^{\prime}$ are fits with the heuristic function Eq. (1).

Table 2

Values of the parameters obtained by fitting the Payne effect curves with the heuristic function in Eq. (1).

\begin{tabular}{lrcc}
\hline & CB & SIL-1 & SIL-2 \\
\hline$P_{0}$ & 7.04 & 4.476 & 4.654 \\
$P_{1}$ & 25.29 & 19.43 & 18.54 \\
$P_{2}$ & 1.39 & 1.57 & 1.65 \\
$P_{3}$ & 0.72 & 0.72 \\
$P_{0}+P_{2}$ & 0.72 & 6.04 & 6.31 \\
$G_{\infty}$ & 8.43 & 1.93 & 2.04 \\
$G_{0}$ & 1.83 & 5.5 & 5.76 \\
\hline
\end{tabular}


The Mullins effect (drop of the modulus after a large amplitude strain cycle) is illustrated in Fig. 5 for the 3 studied materials. Note that all three samples exhibit very similar residual deformation (permanent sets or plasticity). This property may be important in wear studies, in which the sample is submitted to repeated mechanical loadings at amplitudes well beyond the linear regime.

\section{Experimental setup}

\subsection{Rotary tribometer}

The new experimental device (rotary tribometer with in situ optical system) allows: (i) measuring the evolution of the friction coefficient as a function of the number of passages, (ii) measuring the weight loss as a function of the number of passages and (iii) imaging the evolution of wear patterns and determining the typical sizes characterizing it, as a function of the number of passages, in controlled environmental parameters.

Since wear may occur in various situations, there is no standardized definition of wear. Usually, a particular device has to be developed, according to the particular situation to model. The new experimental device that we have developed can be used to recreate and study wear patterns in controlled conditions which model the wear of a tire tread in soft conditions (smooth driving on highways). As mentioned in the introduction, wear is due to slipping which occurs when a point of the tire tread in contact

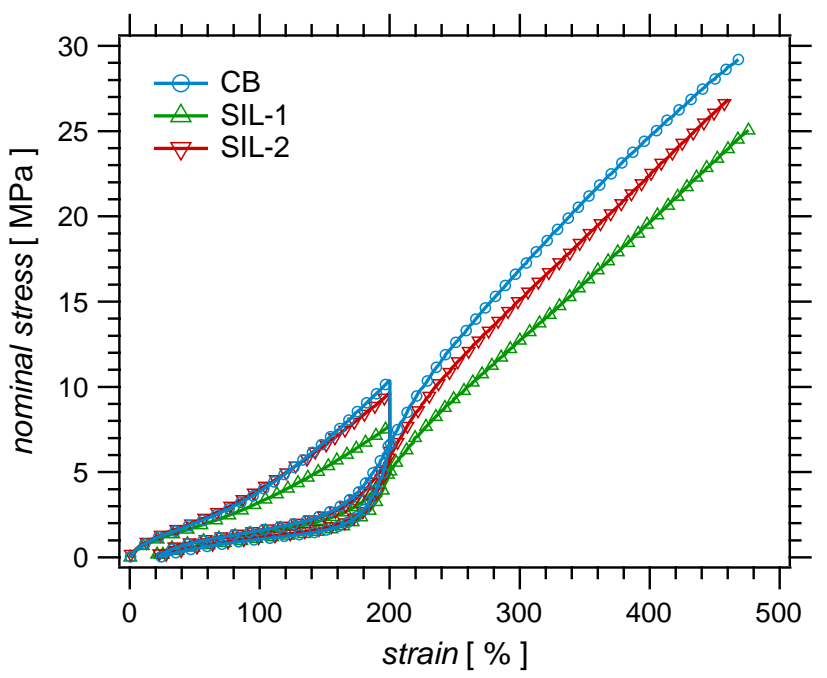

Fig. 5. The nominal stress as a function of the strain after a large amplitude (200\%) tensile cycle (Mullins effect) at room temperature for the 3 studied materials. with the road leaves the contact, thus relaxing the shear stress associated to the traction force transmitted. The general concept of the rotary tribometer is thus to reproduce slipping at the rubber surface, at a sliding velocity comparable to that estimated for a rolling tire tread groove, under a normal pressure comparable to that in a tire tread, and to accumulate a total slipping length comparable to that of a tire. Also, the device must work in open loop mode, i.e. abrasion wastes have to be eliminated between each passage. The major differences between the device developed here and devices already described in the literature are that it works in open loop conditions, and on a relatively thin $(2 \mathrm{~mm})$ test sample in order to increase the contact pressure.

The device is a rotary tribometer working in controlled environment, with an in situ optical observation system. In this section, we describe in details the tribometer. The general scheme of the device is shown in Fig. 6.

The contact between the road surface and the tire tread is represented by a polished stainless steel sphere of diameter $2.54 \mathrm{~cm}$ (one inch) which rubs onto a thin (2 mm thick) plate of the reinforced rubber material glued on a rotating aluminum disk fixed to a motor (motor 2, rotation axis 4 in the general scheme in Fig. 6). The sliding ball (spherical indenter) can rotate thanks to motor 1 (rotation axis 1 in Fig. 6). Motors 1 and 2 are controlled independently. Thus, the contact time and the sliding velocity may be controlled independently. The normal load (from 9 to $250 \mathrm{~N}$ along axis 2 in Fig. 6) is applied manually and measured by a normal load cell. A second load cell measures the tangential load. The mechanical support can translate along axis 3 to locate the sliding ball at a specific position on the sample. The rotation speed of the disk during the sliding passage on the sample, the time between two passages and the number of revolutions are controlled. The rotating disk and the sliding sphere are placed in an oven at controlled temperature (from 0 to $60{ }^{\circ} \mathrm{C}$ ) and humidity. A window has been created for in situ optical observation of the rubbed surface with the optical device (Fig. 6 bottom left). The ratio between tangential and normal loads gives the instantaneous friction coefficient. The tangential and normal load acquisitions are done only in the center zone of the wear track in order to avoid edge effects. 1024 points are acquired and averaged at each passage. In this way, a well-defined value of the friction coefficient all along the wear test is obtained.

During tribological tests, the sliding sphere is cleaned between two passages on the sample by means of an abrasive paper. The abrasive paper is changed to a new piece regularly, depending on the pressure and temperature conditions. The ball is rotated by 10 typically every 400 cycles, in order to keep the surface state roughly constant. At the end of the tests, the sliding sphere is re-polished with three diamond-tipped solutions of 6,3 and $1 \mu \mathrm{m}$ successively. It is then cleaned with acetone, propanol and distilled water.

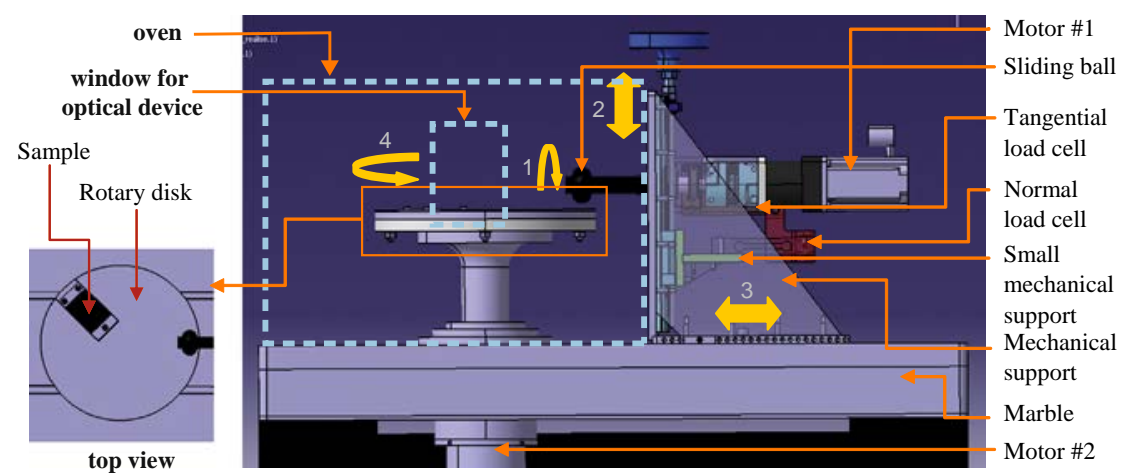

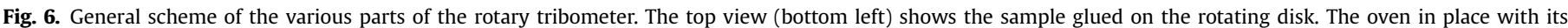
observation window is shown as a dashed box. 
With these procedures, the surface state of the sliding sphere is controlled and maintained constant at the micrometer scale throughout the tests.

Weight losses are measured at regular time intervals during the tests (every 400 passages typically) by weighing the sample. Since elastomer materials are quite sensitive to the environment (hygrometry, temperature, etc.), a reference (unrubbed) sample placed in the same environment as the tested sample is systematically weighed and compared in parallel.

The optical system and the rotary tribometer are both placed on a damped anti-vibration bench.

\subsection{Optical observations}

The obtained wear patterns are observed in situ through an optical system (Fig. 7) in order to obtain general information on the wear surface topography, the type of wear pattern (ridges, craters, grooves and rollers) and their dimensions (width and depth). The optical system is an angle tilted optical pen (commercialized by STIL), which operates in a way similar to a confocal laser scanning microscope (white light). A mechanical device has been developed to adapt the optical system to the rotary tribometer. The displacement of the optical system is performed with motorized tables in the $(x, y)$ plane (millimeter scale) and manually in direction $z$ (micrometer scale). The mounting of the optical device is shown in Fig. 9. It allows to acquire topographic images with millimeter or micrometer scale resolution in the $(x, y)$ plane and from micrometer to nanometer scale resolution in depth direction $z$.

Complementary to in situ optical observation, wear surfaces and profiles have been characterized by Scanning Electron Microscopy (SEM). By cutting the samples perpendicular to the rubbed surface, the wear pattern profiles, as well as the state of the material at various depth beneath the surface, can be characterized in directions parallel or perpendicular to the sliding direction. Cuttings are performed by cryo-microtomy and the observed surfaces are coated with a $2 \mathrm{~nm}$ thick platinum layer.

\subsection{Description of the contact in indentation}

For a tire tread in usage conditions, the contact dimension is close to or larger than the sample thickness, which implies that the sample (the part of it close to the surface) may be submitted to some degree of isotropic compression in addition to uniaxial compression. Thus, in order to mimic the contact in a realistic way by using a spherical indenter, it is thus essential to work in conditions in which the dimension of the contact zone is close to or larger than the elastomer sample thickness. In this way, the pressure, and specifically the normal pressure, may be increased while keeping relatively small local indentation amplitudes. Indeed, uniaxial compression corresponds to a modulus of order $10^{7} \mathrm{~Pa}$ (see Fig. 2), while isotropic compression corresponds to a

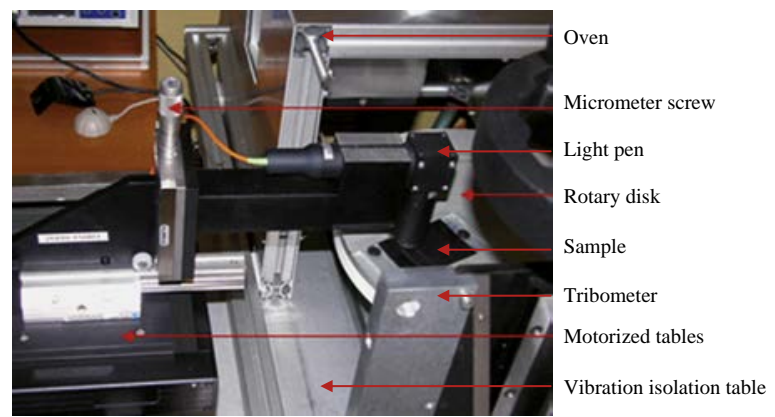

Fig. 7. Scheme of the in-situ optical observation system. bulk modulus of the order $10^{9} \mathrm{~Pa}$. In this configuration, an increasingly large pressure may be applied as the sample gets thinner (compared to the indenter diameter).

Indentation curves (normal force vs. indentation) have been measured and are shown in Fig. 8 for the three samples described in Section 2.1. Different regimes may be distinguished in these curves, and a significant upward inflexion of the curves is effectively
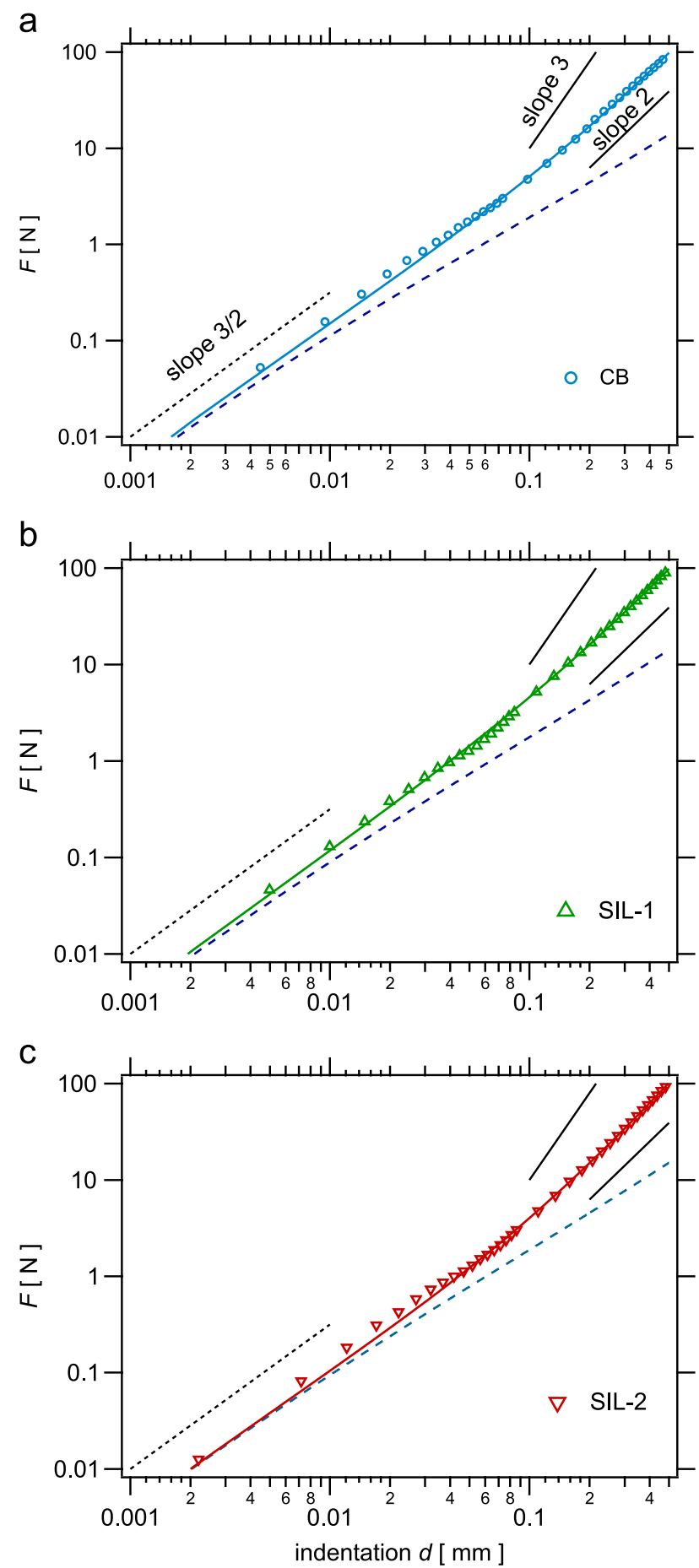

Fig. 8. Normal force vs. indentation depth for the three samples: (a) CB; (b) SIL-1; (c) SIL-2. Symbols are experimental points; dashed curves correspond to the Hertz formula (Eq. (2)) including a non-linear variation of the modulus as shown in Fig. 4. Full curves are fits of the experimental curves with Eq. (8), including the non-linear variation of the modulus. The various power laws are indicated (dots: Hertz, Eq. (2); full lines: Eqs. (5) and (6)). 
observed beyond $0.1 \mathrm{~mm}$ in indentation or equivalently beyond 7$10 \mathrm{~N}$ in force.

These curves may be analyzed as follows. The indentation of a sample in the form of an incompressible layer coated on a more rigid substrate has been described in details already [43-45]. However, only linear materials were considered and friction at the interface was not considered. We propose here an interpolation method which allows to deal with materials which have a non-linear (hyperelastic) constitutive law.

For a spherical indenter of radius $R$ acting on a semi infinite medium of Young's modulus $E$, in linear elasticity and for a frictionless contact, the relationship between the normal force $F_{n}$ and the indentation $\delta_{\infty}$ (where the subscript ${ }_{\infty}$ stands for a semiinfinite medium) is given by Hertz's formula [26]:

$F_{n}=\frac{4}{3} E^{*} R^{1 / 2} \delta_{\infty}^{3 / 2}$

where the effective modulus $E^{*}$ is defined as (for an incompressible material with Poisson coefficient $\nu=0.5$ ):

$E^{*}=\frac{E^{\prime}}{1-\nu^{2}}=\frac{4 E}{3}=4 G^{\prime}$

where $G^{\prime}$ is the shear modulus. Then the contact radius as a function of the normal load is given by

$a_{\infty}=\left(\frac{3 F_{n} R}{4 E^{*}}\right)^{1 / 3}$

For a thin layer of thickness e glued on a rigid substrate (Fig. 9), the force vs. indentation relationship for a spherical indenter with frictionless contact may be expressed by the scaling relationship [46]:

$F_{n} \approx \frac{E^{*} R^{2} \delta^{3}}{e^{3}}$

Friction inhibits lateral displacements at the contact. In this case, the situation should be better described by the elastic layer model, with no lateral displacement, which obeys the relationship [26]:

$F_{n} \approx \frac{E^{*} R \delta^{2}}{1-2 \nu e}$

For $\nu \approx 0.5$, the quantity $K=E^{*} /(1-2 \nu$ ) (of the order of the bulk (compressibility) modulus) is much larger than $E^{*}$. In this situation, the normal compression force indeed increases as the ratio $R / e$ increases at a given indentation value. The contact radius is given by $a^{2} \approx \delta R$. The indentation may be described by the dimensionless parameter $\delta R / e^{2}$, which is of the order $(a / e)^{2}$. In our experimental conditions, the contact radius (estimated from the width of the wear track) is of the order $2 \mathrm{~mm}$, i.e. of the same order of magnitude as the sample thickness $e$, and the parameter $\delta R / e^{2}$ is of order one. To interpolate between the two regimes described above (Eqs. (2) and (5) or (6)), we propose an heuristic analytical approach in which the ratio $\delta / \delta_{\infty}$, used as the

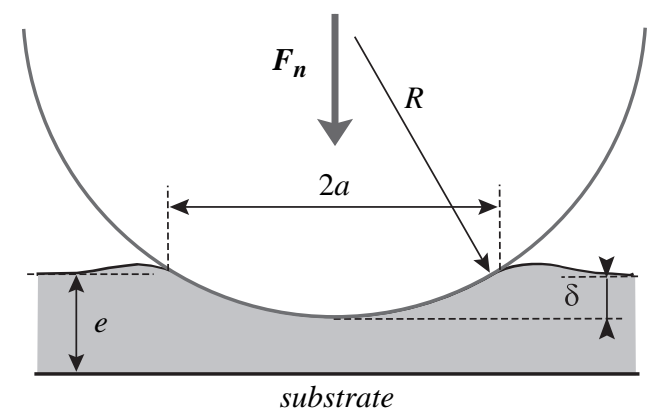

Fig. 9. Schematics of the indentation of a layer of thickness $e$ glued on a substrate by a spherical indenter of diameter $2 R$. interpolated parameter (since it is $\delta$ which is measured in our experimental setup), is expressed as a function of the dimensionless parameter $\delta R / e^{2}$ as follows:

$$
\left(\delta / \delta_{\infty}\right)^{3 / 2}=\frac{1}{1+\phi_{1}\left(\frac{\delta R}{e^{2}}\right)^{1 / 2}+\phi_{2}\left(\frac{\delta R}{e^{2}}\right)+\phi_{3}\left(\frac{\delta R}{e^{2}}\right)^{3 / 2}}
$$

The normal load may be expressed dimensionally as $F_{n} \approx \delta_{\infty}^{-3 / 2} F_{n} \delta^{3 / 2}\left(\delta / \delta_{\infty}\right)^{-3 / 2} \approx E R^{1 / 2} \delta^{3 / 2}\left(\delta / \delta_{\infty}\right)^{-3 / 2} \quad$ (see Eq. (2)), which, from Eq. (7), gives the following load vs. indentation interpolation relationship:

$\left.F_{n}=\phi_{0} E^{*} \delta^{3 / 2} R^{1 / 2} 1+\phi_{1}\left(\frac{\delta R}{e^{2}}\right)^{1 / 2}+\phi_{2}\left(\frac{\delta R}{e^{2}}\right)+\phi_{3}\left(\frac{\delta R}{e^{2}}\right)^{3 / 2}\right)$

with $\phi_{0}=4 / 3$ in the Hertz contact approximation (see Eq. (2)). Eq. (2) is recovered in the limit $\delta R / e^{2} \ll 1$ (Hertz contact) and Eq. (5) or (6) are recovered in the opposite limit $\delta R / e^{2} \gg 1$ (infinitely thin layer), according to the values of the $\phi_{1}, \phi_{2}$ and $\phi_{3}$ parameters.

For the curves in Fig. 8, at small values of the force, as the contact radius, and thus the extension of the strain zone, are small compared to the thickness $e$, a Herz regime, with $F \approx \delta^{3 / 2}$ (Eq. (2)), is effectively observed.

At intermediate force values, there is a slight decrease of the measured force relative to the Herz formula. This downward inflexion of the force occurs for indentation values of the order $0.02-0.1 \mathrm{~mm}$, that is average deformations of roughly $\delta / e \approx 0.01-$ 0.05 (1-5\%). This corresponds exactly to the Payne effect regime, in which the modulus decreases as the strain amplitude increases (see Fig. 4). Note that part of this relative decrease may also be due to the onset of slippage in the contact area.

At even larger force values, a third regime is observed, in which the curves seem to show an apparent power law very close to $F \approx \delta^{2}$, compatible with the confined elastic thin layer model (Eq. (6)).

The experimental indentation curves have been fitted with Eq. (8), with coefficient $\phi_{0}$ fixed to 1.33 (corresponding to the Hertz model) and Eq. (1) used to account for the non-linear variation of the modulus. Coefficient $\phi_{3}$ was fixed to zero, corresponding to the observed exponent in the third regime of the curves. Best fits are shown in Fig. 8 (full curves). The obtained values of the coefficients $\phi_{1}$ and $\phi_{2}$ are summarized in Table 3 . Note that the modulus values measured independently allow matching the curves quantitatively at small indentations values.

The ratio $\delta / \delta_{\infty}$ is plotted as a function of the dimensionless quantity $\delta R / e^{2}$ in Fig. 10. Symbols are obtained from the experimental curves and solid curves correspond to Eq. (7), with the same values of the coefficients $\phi_{1}, \phi_{2}$ and $\phi_{3}$ as determined from fitting the curves in Fig. 8. This graph shows that the proposed approximate relationship (Eq. (8)) gives a good fitting of the indentation data roughly over roughly one decade, from $\delta R / e^{2} \approx 0.2$ up to $\delta R / e^{2} \approx 2$, which is precisely the range of interest in the wear tests performed here. The non-monotonic variation of $\delta / \delta_{\infty}$ may be due to onset of partial slippage within the contact zone, which would partially relax some stress and thus allow $\delta / \delta_{\infty}$ to increase abruptly at the corresponding value of $\delta$.

Table 3

Values of the parameters obtained by fitting the indentation curves with Eq. (8).

\begin{tabular}{lllr}
\hline & CB & SIL-1 & SIL-2 \\
\hline$\phi_{0}$ & 1.33 & 1.33 & 1.33 \\
$\phi_{1}$ & 1.44 & 1.33 & -0.03 \\
$\phi_{2}$ & 2.67 & 2.67 & 3.65 \\
$\phi_{3}$ & 0 & 0 & 0 \\
\hline
\end{tabular}




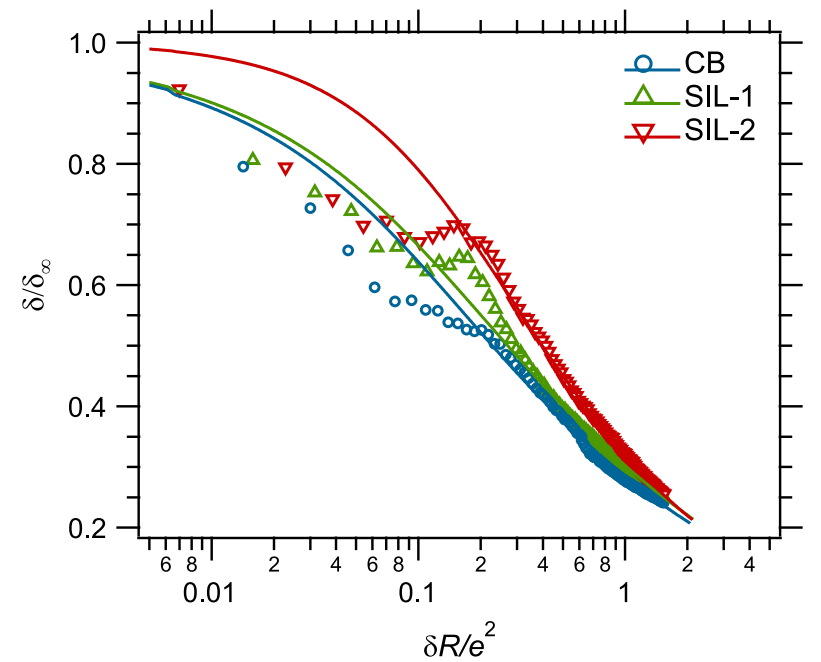

Fig. 10. The ratio $\delta / \delta_{\infty}$ as a function of the dimensionless quantity $\delta R / e^{2}$ for the 3 samples. Symbols are obtained from the experimental curves and solid curves correspond to Eq. (7) with the same values of the coefficients $\phi_{1}, \phi_{2}$ and $\phi_{3}$ as determined from fitting the curves in Fig. 8.

Finally, we have shown and discussed quantitatively how working on a thin sample coated on a rigid substrate allows increasing the normal force (or contact pressure). However, the apparent modulus in this regime is not very much higher than at smaller forces, as would be expected from Eq. (6). Thus, one does not yet reach a regime in which the confinement, i.e. the effect of the bulk (compressibility) modulus would become predominant. In a range of indentation of the order $0.2 \mathrm{~mm}$, the force is increased typically by a factor of about 4 . This geometry potentially gives an additional control parameter, the ratio of the indenter radius $R$ to the sample thickness $e$, to modify the loading conditions of the system.

The average pressure $P=F_{n} / \pi a^{2}$ and the contact radius may be deduced from indentation curves. For instance, for a two millimeter thick sample and an applied normal force $9.4 \mathrm{~N}$, the pressure actually applied on the contact zone is close to $1.5 \mathrm{MPa}$.

\section{Results of wear tests and discussion}

Tribological tests have been performed on the three materials described in Section 2.1, using the tribometer described in Section 3.1. These samples are representative of real materials used in tire treads. In order to insure that applied test conditions are representative of real condition, orders of magnitude as regards rolling and sliding in the contact zone in standard usage conditions of a tire must be determined.

As a result of friction, the tread sticks to the road in most of the contact zone (supposed to be a rectangle of dimensions $2 a$ in the rolling direction and $l=0.35 \mathrm{~m}$ (the width of the tread) in the transverse direction). Due to compression of the tire, the material in the contact zone is submitted to tangential strain, which can be estimated from the difference between the arc and the chord of the tread in the contact zone (see Fig. 11). The strain must be continuous, hence zero, at the leading edge, where the rubber material enters into the contact zone, and is maximum at the trailing edge. As a result, tangential stress develops, which leads to slip at the trailing edge, as the local tangential stress would become larger than $\mu p$, where $\mu$ is the friction coefficient and $p$ the local normal pressure (as schematized in Fig. 11). In real conditions, lateral tire slip during direction changes (as well as perhaps during strong deceleration phases) comes in addition to this single direction slippage.

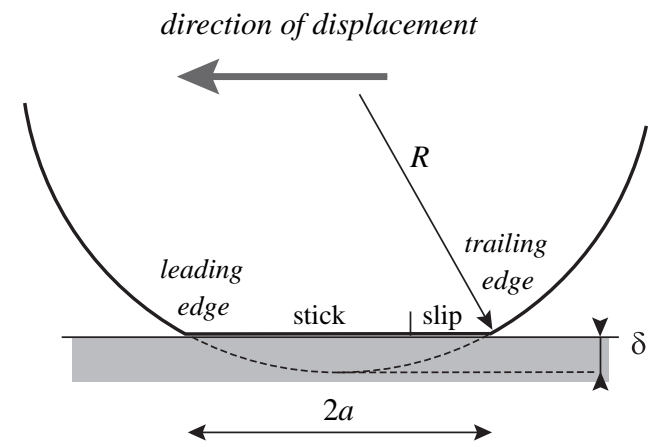

Fig. 11. Schematics of the contact zone of a tire tread groove. Slip occurs at the trailing edge of the contact zone, where the tangential over normal stress ratio becomes larger than the friction coefficient $\mu$.

The average normal pressure applied at the interface between the tread and the road, which corresponds to the supported weight per tire divided by the contact area, is the tire pressure. A normal pressure of the order $P_{0} \approx 0.8 \times 10^{6} \mathrm{~Pa}$ (for a truck) and a normal force of the order $2.5 \times 10^{4} \mathrm{~N}$ are applied on each tire, which results in a width of the contact area $2 a \approx 0.1 \mathrm{~m}$ and a deformed height $\delta \approx 2.5 \mathrm{~mm}$ (see Fig. 11). The average deformation of the tire tread is of the order $10 \%$ (except perhaps very close to the contact, due to road asperities). In the present tests, the normal load was varied between $9.4 \mathrm{~N}$ and about $30 \mathrm{~N}$, which indeed corresponds to an average normal pressure of order $0.8-$ 1.7 MPa with a diameter of the contact zone of typically 3 to $4 \mathrm{~mm}$.

The slip length $u$ is the difference between the arc and the chord in the contact zone (Fig. 11), which gives

$u \approx \frac{a^{3}}{3 R^{2}}$

With $a \approx 0.05 \mathrm{~m}$ and $R=0.5 \mathrm{~m}$, this gives a slip length $u \approx 0.17 \mathrm{~mm}$. At a velocity $V \approx 30 \mathrm{~ms}^{-1}(90 \mathrm{~km} / \mathrm{h})$, the contact time of any point of the tread $t_{c}=2 a / V$ is of the order $t_{c} \approx 3 \times 10^{-3} \mathrm{~s}$. The order of magnitude of the slipping velocity is $v_{s} \approx u / t_{c}$, which gives $v_{s} \approx 6 \times 10^{-2} \mathrm{~ms}^{-1}$. In the test, the sliding velocity has been chosen to be $10 \mathrm{~mm} \mathrm{~s}^{-1}$, with no rotation of the sliding sphere. Note that the sliding velocity may be further increased independently of other parameters by counter-rotating the sphere.

The strain rate within the material close to the contact may be estimated as follows. The tangential stress $\sigma_{x}$ at the surface (at onset of slipping) is of order $\mu P_{0}$. It is equal to the shear stress in the material in contact with the surface. Hence the shear strain $\epsilon_{x} \approx \mu P_{0} / G^{\prime}$, where $G^{\prime}$ is the shear modulus of the material, and the shear rate:

$\dot{\epsilon}_{x} \approx \frac{\epsilon_{X}}{t_{c}} \approx \frac{\mu P_{0} V}{2 a G^{\prime}}$

For a shear modulus of the order $3 \times 10^{6} \mathrm{~Pa}$ and a friction coefficient of the order 2 (see later), the shear rate is found to be of order $160 \mathrm{~s}^{-1}$.

Thus, the contact between a point of a truck tire tread and the road is characterized by a periodic solicitation of frequency $f \approx V / 2 \pi R \approx 10 \mathrm{~Hz}$, a contact time $t_{c} \approx 3 \times 10^{-3} \mathrm{~s}$ and a maximum shear rate $\dot{\epsilon}_{x} \approx 100 \mathrm{~s}^{-1}$.

The lifetime of a tire tread is of the order $10^{5}$ driven kilometers, which corresponds to about $3 \times 10^{7}$ cycles, and thus a total slip length of about $5 \times 10^{3} \mathrm{~m}$, assuming that a point of the tread is solicitated at each cycle. During his lifetime a truck tire tread looses about $13 \mathrm{~mm}$ in height by abrasion, which gives an average of about $4 \times 10^{-10} \mathrm{~m}$ per cycle. Thus, the wear is roughly of the order $2.5 \times 10^{-6} \mathrm{~m}$ of abrased height per slip meter. In our tests, 
each passage corresponds to a slipping length of about $4 \mathrm{~mm}$ (the diameter of the contact zone). 10000 passages are performed typically, which thus give a total slipping length of about $40 \mathrm{~m}$ and should produce an abrased height of about $100 \mu \mathrm{m}$, which indeed corresponds roughly to the observed orders of magnitude (see Figs. 14b, 15b and 16b).

Note again that the above considerations apply to slippage in steady driving conditions, independent of lateral slippage which further increases abrasion. The above estimate would therefore represent a lower limit of tire tread wear in ideal conditions.

To summarize, the following conditions have been applied for the tests shown here: sliding velocity: $10 \mathrm{~mm} \mathrm{~s}^{-1}$ (with no rotation of the sliding sphere); normal load: 9.4-30 N; number of passages: 3000, 6000 and 10000 ; time delay between two passages: $20 \mathrm{~s}$; temperature $25{ }^{\circ} \mathrm{C}$; hygrometry $\approx 50 \%$.

We shall describe typical wear patterns obtained in the three studied materials, emphasizing the differences observed when materials parameters are changed, even though overall properties of the materials are quite similar anyway, since we have chosen to characterize representative materials.

\subsection{Effect of the type of fillers}

Natural rubber materials reinforced by carbon black fillers (CB) and by silica fillers (SIL-1) are compared in this section. Average friction coefficients for the CB and SIL- 1 samples are plotted in Fig. 12 as a function of the number of passages (cycles). Both curves show the same qualitative behavior: the average friction coefficient first tends to increase during the first 1000 passages, then, after reaching a maximum, it decreases slowly and finally reaches a stable value after typically 4000 (or a few thousands) passages. In this final steady state, the average values for samples CB and SIL-1 are close to 1.8 and 1.5 , respectively. The consistently higher value observed in the carbon-black filled sample (CB) than in SIL-1 may be attributed to the fact that this sample is more dissipative than the silica filled samples, with a larger Payne effect amplitude (see Fig. 4).

The overall weight loss curves are plotted as a function of the passages number for the two samples CB and SIL-1 in Fig. 13. After some transient regime (of the order of a few $100 \mathrm{~s}$ of passages), the curves are roughly linear, which allows to define a wear rate, or weight loss per cycle (the slope of the curves in Fig. 13). Both samples have nearly the same wear rate, of the order $1 \times 10^{-6} \mathrm{~g}$ per passage. Thus, after 10000 passages, the overall weight loss is

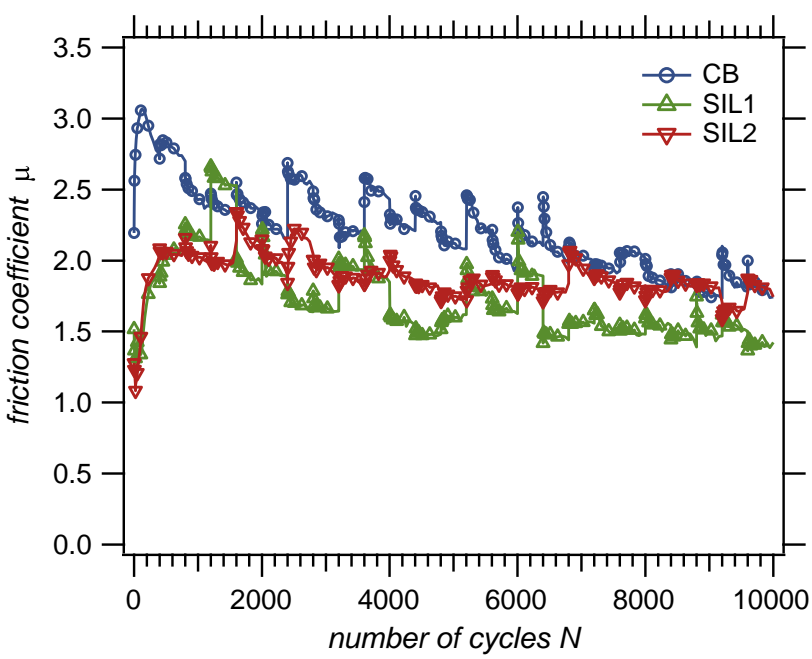

Fig. 12. Friction coefficients as a function of the number of passages for the three samples CB, SIL-1 and SIL-2, under normal load $9.4 \mathrm{~N}$ and at room temperature.

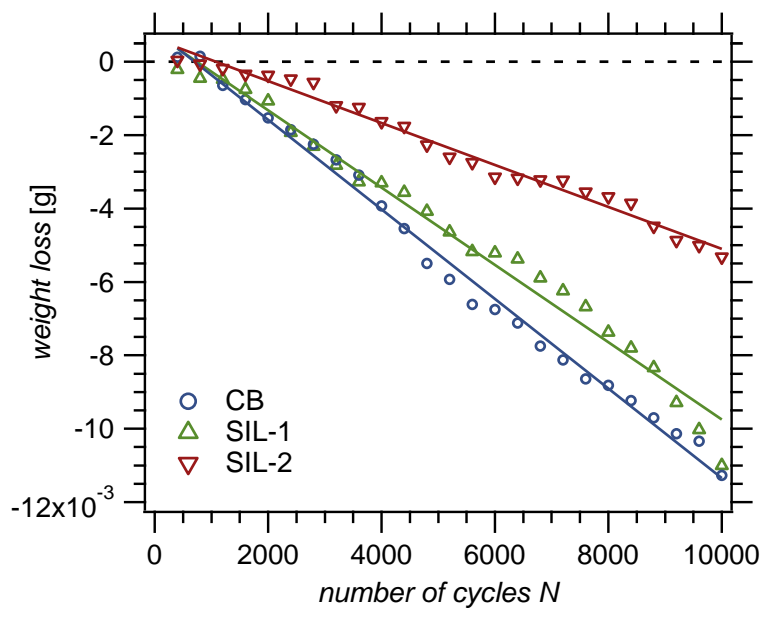

Fig. 13. Weight loss as a function of the number of passages for the three samples CB, SIL-1 and SIL-2, under normal load $9.4 \mathrm{~N}$ and at room temperature.

approximatively $1.1 \times 10^{-2} \mathrm{~g}$ for the CB sample and $1 \times 10^{-2} \mathrm{~g}$ for the SIL-1 sample.

The wear patterns obtained on the CB and SIL- 1 samples after 10000 passages are shown in Figs. 14 and 15 respectively, observed first with the in-situ optical system (see Section 3.2) (Figs. 14a and 15a) and then in Scanning Electron Microscopy (SEM) parallel to the sliding direction at the center of the wear track (14c and 15c).

Ridges are present in both CB and SIL-1 samples (Figs. 14a and $15 a)$. Ridges are oriented perpendicularly to the sliding direction and form roughly parabolic patterns. Ridges are larger for the SIL-1 than for the CB sample as regards both the average longitudinal distance and height (Figs. 14c and 15c).

It is coherent with the tearing out of material at the contactback with the sliding ball.

Figs. 14b and 15b correspond to the average topographic profile of images along the wear track, obtained with the in-situ optical system (Figs. 14a and 15a respectively). They show that the depth and the width of wear patterns are larger for sample SIL-1 than for sample CB. Indeed, for 10000 passages and for the two samples CB and SIL-1, the widths of wear pattern are respectively $4 \mathrm{~mm}$ and $4.5 \mathrm{~mm}$ and the depth are $20-25 \mu \mathrm{m}$ and $150 \mu \mathrm{m}$.

From the topographic profiles measured optically (depth and width of the wear patterns (Figs. 14b and 15b), the weight loss of the samples may in principle be estimated from the worn volume. Losses estimated in this way (Figs. 14 and 15b) are only semiquantitatively coherent with the overall weight losses (Fig. 13). Differences between these two estimates may be due to the difficulty in defining the baseline in the patterns shown in Figs. $14 \mathrm{~b}$ and $15 \mathrm{~b}$.

To summarize the observations, substituting carbon black by silica fillers (at same filler volume fraction, matrix glass transition temperature and hardness) leads to slight differences in the wear behavior. Both materials have similar wear rates and exhibit wear ridges. However, the wear patterns are quite different. Ridges are larger in the SIL-1 than in the CB material. The difference in wear behavior might be correlated to the difference in ultimate properties of these materials (see Fig. 3). Indeed, CB has a more pronounced stress upturn at high strain values (or a slightly smaller ultimate strain), which may explain smaller ridges in $\mathrm{CB}$ than in SIL-1.

\subsection{Effect of the silica fraction}

In this section, NR samples reinforced with $50 \mathrm{phr}$ (SIL-1) and $55 \mathrm{phr}$ (SIL-2) of silica are compared. Average friction coefficients 

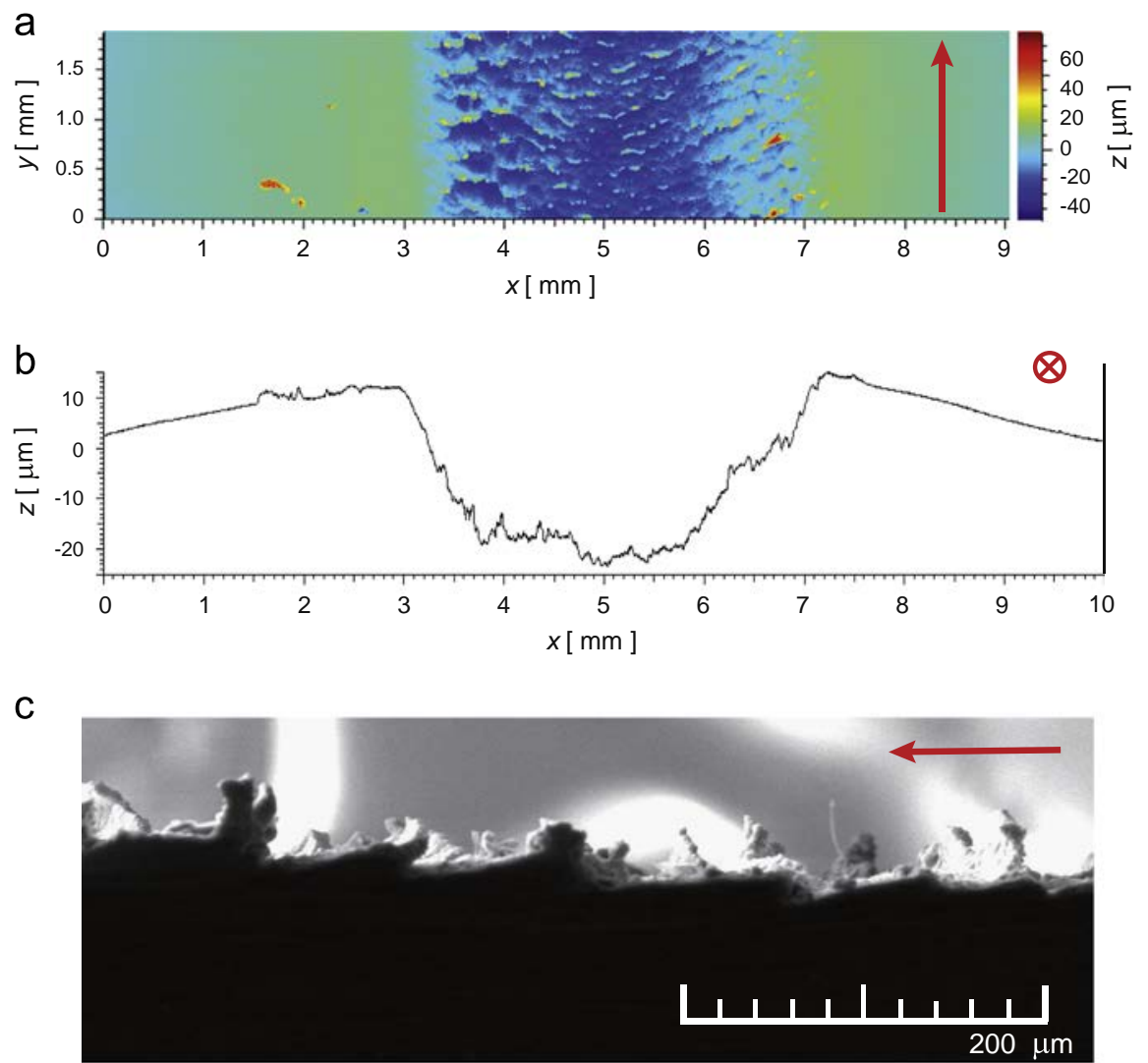

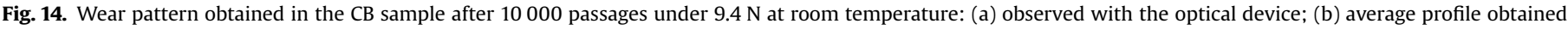

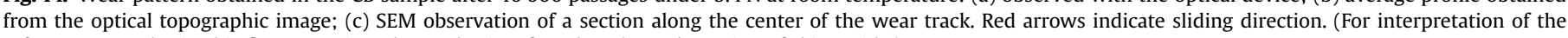
references to color in this figure caption, the reader is referred to the web version of this article.)

for the SIL- 1 and SIL-2 samples are plotted in Fig. 12 as a function of the number of cycles. Again, both curves show the same qualitative behavior: the average friction coefficient is larger and tends to increase during the first 1000 passages, then it decreases slowly and finally reaches a stable value after a few thousand passages. In this final steady state, the average values for the SIL-1 and SIL-2 samples are close to 1.5 and 2, respectively. A consistently higher value is observed in the more reinforced sample SIL2 as compared to SIL-1.

Weight loss curves are plotted as a function of the passages number for the SIL-1 and SIL-2 samples in Fig. 13. The wear rate of SIL-2 $\left(6 \times 10^{-7} \mathrm{~g} /\right.$ passage $)$ is about twice smaller than that of SIL-1 $\left(1 \times 10^{-6} \mathrm{~g} /\right.$ passage $)$. Correlatively, the overall weight loss after 10000 passages is approximatively $1 \times 10^{-2} \mathrm{~g}$ for SIL- 1 and $5 \times$ $10^{-3} \mathrm{~g}$ for SIL-2.

As previously, the same observations have been performed for SIL-2 after 10000 passages, first with the in-situ optical system (Fig. 16a and b), and then with Scanning Electron Microscopy (SEM) (Fig. 16c) on sections parallel to the sliding direction in the center of the wear track.

The wear patterns observed in the two materials are clearly different. When comparing Figs. 14a and 16a, SIL-1 exhibits well established ridges whereas SIL-2 shows craters.

The average topographic profiles after 10000 passages are clearly different in SIL-1 and SIL-2. The wear pattern widths are respectively $4.5 \mathrm{~mm}$ and $4 \mathrm{~mm}$ and depths are $150 \mu \mathrm{m}$ and 20-25 $\mu \mathrm{m}$.

The weight loss estimated by calculation of volume loss from the values of depth and width of the wear pattern profile (Fig. 16b) is semi-quantitatively coherent with those measured by directly weighing the sample (Fig. 13).
To summarize the observations, the weight loss is significantly reduced in the SIL-2 material as compared to SIL-1. Thus, the filler amount has a significant impact on the wear resistance of reinforced natural rubber materials. On one hand, optical and SEM observations confirm this idea: the wear pattern is much more pronounced in material SIL-1 (with well developed ridges) than in material SIL-2 (which has almost no ridge yet after 10000 passages). On the other hand, friction coefficients are different for these two materials SIL-1 and SIL-2. Thus it seems that some correlation exists between the friction coefficient and the wear pattern. Moreover, mechanical data are slightly different for these two materials, SIL-2 being harder and more reinforced (see Fig. 3).

\subsection{Effect of the normal load and temperature}

The wear map of material SIL-2 as a function of the number of cycles and normal load (between 9.4 and $30 \mathrm{~N}$ ) is shown in Fig. 17 at two different temperatures. Note that, due to self-heating of the material in actual usage, the temperature $60{ }^{\circ} \mathrm{C}$ is close to real operating conditions. The apparent contact pressure reported in Fig. 17 is the average pressure obtained by dividing the normal load by the apparent contact area, as estimated from the width of the wear track. A transitory pattern (FT) is observed at numbers of cycles smaller than roughly 1500 , independently of the applied load. It shows small rollers perpendicular to the sliding direction. This latency period corresponds to removing the skin layer (due to processing) and accommodation of the material surface. Then, at room temperature $\left(25^{\circ} \mathrm{C}\right)$, two main regions can be distinguished: craters at small load values and ridges at higher load, with a transition from craters to ridges shifting to lower load values as the number of cycles increases. At high temperature $\left(60^{\circ} \mathrm{C}\right)$, wear 


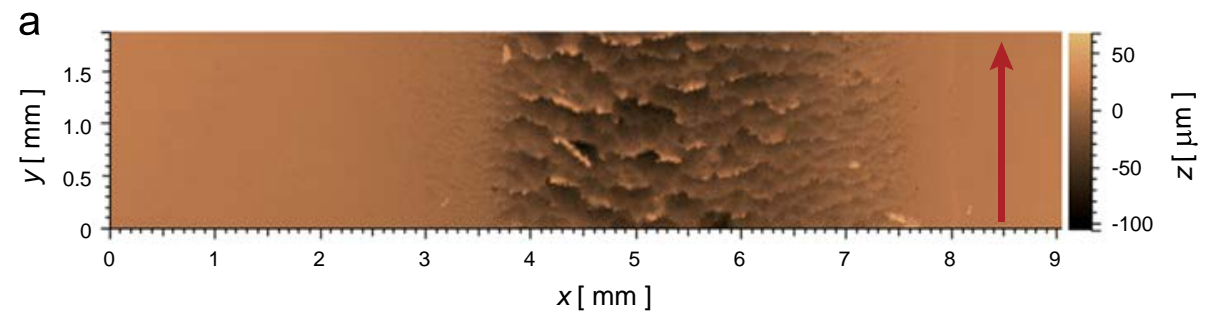

$\frac{E}{\frac{E}{\Xi}}$
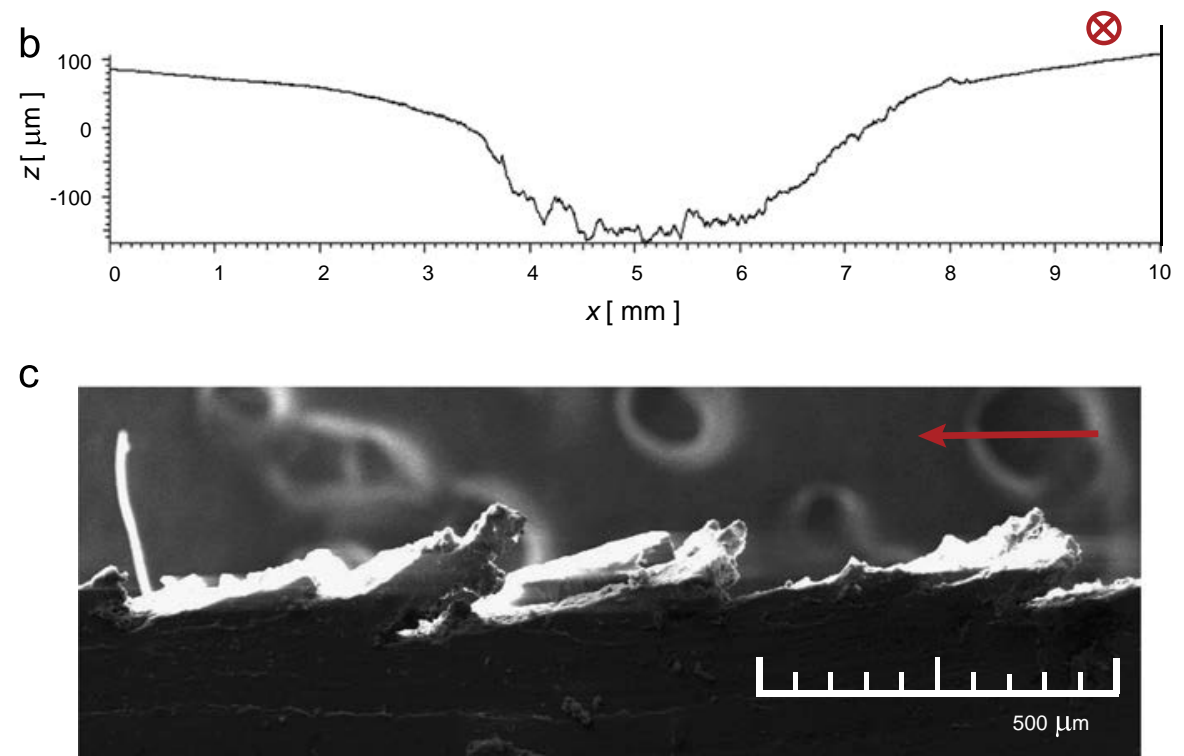

Fig. 15. Same as Fig. 14 for the SIL-1 sample, after 10000 passages under $9.4 \mathrm{~N}$ at room temperature: (a) wear pattern observed with the optical device; (b) average profile; (c) SEM observation of a section along the center of the wear track. Red arrows indicate sliding direction. (For interpretation of the references to color in this figure caption, the reader is referred to the web version of this article.)
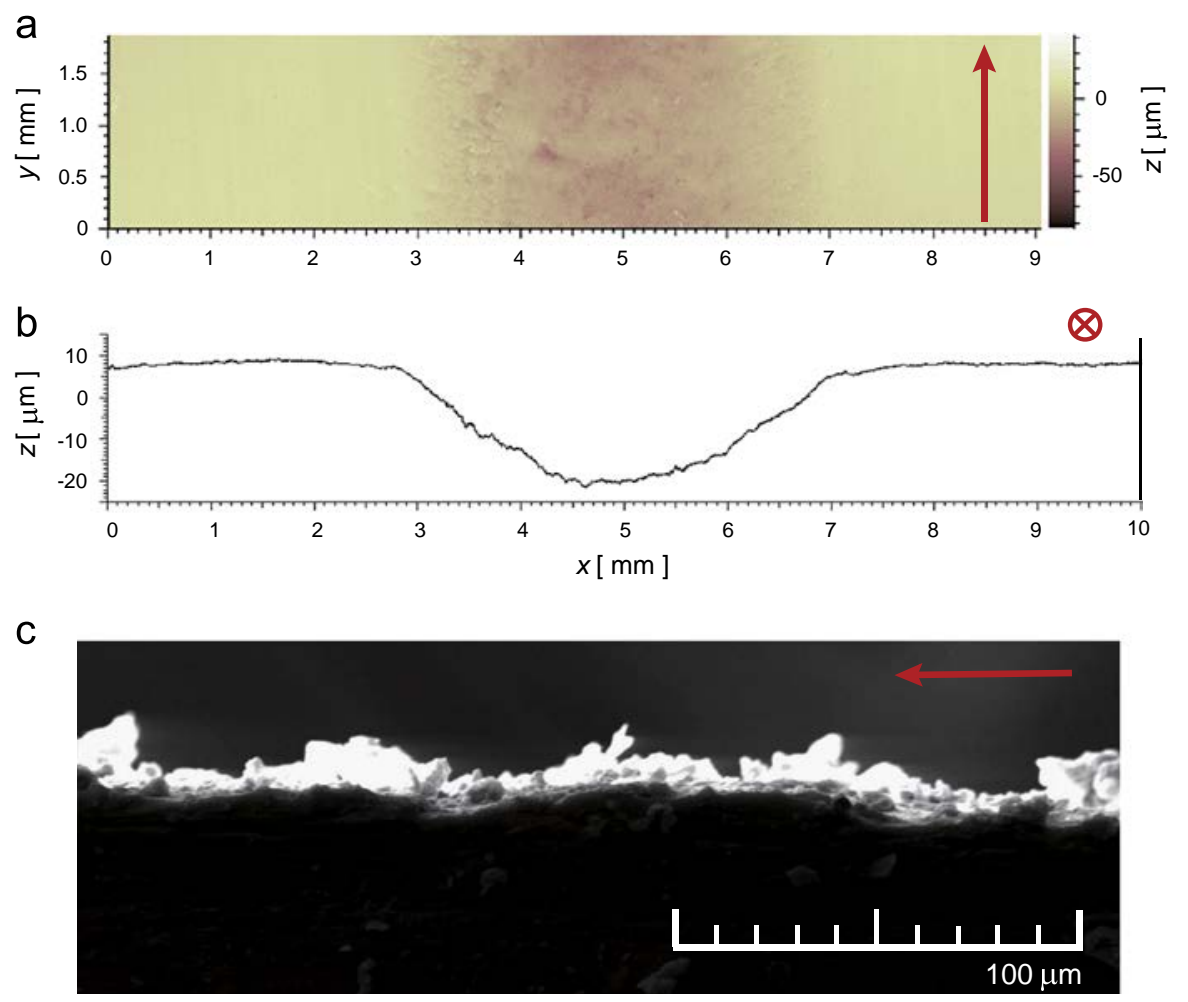

Fig. 16. Wear pattern obtained in the SIL-2 sample after 10000 passages under $9.4 \mathrm{~N}$ at room temperature: (a) observed with the optical device; (b) average profile; (c) SEM observation of a section along the center of the pattern. Red arrows indicate the sliding direction (as in Figs. 14 and 15). (For interpretation of the references to color in this figure caption, the reader is referred to the web version of this article.) 


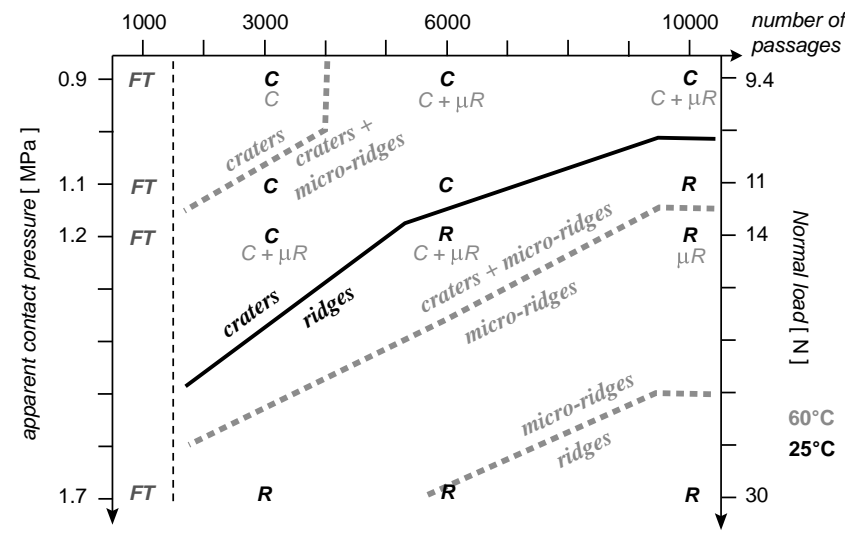

Fig. 17. Wear pattern map for the SIL-2 sample as a function of the number of passages, the normal load and the apparent contact pressure, at temperatures $25^{\circ} \mathrm{C}$ and $60^{\circ} \mathrm{C}$. Black $\left(25^{\circ} \mathrm{C}\right)$ or gray $\left(60^{\circ} \mathrm{C}\right)$ letters correspond to all investigated points: FT: transient pattern; C: craters; R: ridges; $\mu \mathrm{R}$ : micro-ridges. Boundaries separating the different pattern regions are indicated by grey dashed curves $\left(60{ }^{\circ} \mathrm{C}\right)$ or black full curves $\left(25^{\circ} \mathrm{C}\right)$.

patterns are smoother, with micro-ridges coexisting with craters and evolving towards ridges as the load increases.

\subsection{Discussion}

The three studied materials show clean, well-developed ridge patterns at room temperature, after some transitory regime necessary for establishing a steady state. This is in agreement with previous observations of similar ridge patterns obtained in blade abrasion tests [47]. The transitory regime observed in [47] was of the order $10^{3}$ cycles, as in the present work. This demonstrates that there is some generic mechanism leading to similar ridge patterns in the steady state. We attribute this generic process to the stick-slip mechanisms related to the development of strong tangential strain at the interface. These lateral strains develop in an intrinsic way due to the overall deformation of tire tread, independent of possible lateral slip motions. The present results suggest that this generic mechanism is correctly implemented in our device.

As regards the difference in abrasion wear of the various studied materials, it was inferred in [48] that creating larger asperities (higher ridges) during abrasion results in larger wear rates, and that weaker materials (in the sense of faster fatigue crack propagation under similar conditions) develop smaller asperities, thus being more abrasion-resistant. In our case, we compare only reinforced materials. Material SIL-2, which is the most reinforced one, have both smaller ridges and lower abrasion weight loss. Thus, changing the type of filler may affect the abrasion behavior independently of the toughness or resistance to fatigue crack propagation.

\section{Conclusion}

We have developed a new tribometer with in situ optical observation, based on a sliding indenter which allows to mimic the wear of tire treads in soft driving conditions, in laboratory conditions under controlled environment. Several relevant observables can be determined as a function of the number of passages, of the applied load in the contact and of environmental parameters (mainly temperature): (i) the friction coefficient, (ii) the weight loss and (iii) wear pattern topographies.

Preliminary tribological tests have been carried out on three different reinforced natural rubber materials filled with silica or carbon black (materials CB, SIL- 1 and SIL-2). The tribological tests realized for each sample at 3000, 6000 and 10000 passages show the good reproducibility of these tests and results. Moreover, results presented in the last section show the panel of possibilities of the new experimental device: these data are essential in order to understand the wear of reinforced natural rubber. Wear mechanisms will be studied in more details in a forthcoming paper.

The first results obtained on reinforced natural rubber materials show qualitatively the impact of the filler type and the fillers fraction. These preliminary results show that it is possible to formulate silica-reinforced natural rubber samples which have a wear resistance similar to, or perhaps even better than, similar carbon black-reinforced natural rubber samples.

\section{References}

[1] G. Petitet, Contribution à la compréhension des mécanismes élémentaires d'usure douce des élastomères chargés réticulés, Ph.D. Thesis, École Centrale de Lyon, 2003.

[2] E. Genin-Blanchard, Étude des mécanismes d'usure élémentaire des élastomères chargés réticulés, Ph.D. Thesis, École Centrale de Lyon, 2006.

[3] G. Petitet, M. Barquins, Matériaux caoutchoutiques - Morphologie, formulation, adhérence, glissance et usure, ed. p.p.e.u. romandes, 2008.

[4] A.G. Veith, A review of important factors affecting treadwear, Rubber Chemistry and Technology 65 (1991) 601-659.

[5] K.A. Grosch, Rubber friction and its relation to tire traction, Rubber Chemistry and Technology 80 (2007) 379-411.

[6] A.H. Muhr, A.D. Roberts, Rubber abrasion and wear, Wear 158 (1992) 213-228.

[7] K.A. Grosch, A. Schallamach, Relation between abrasion and strength of rubber, Rubber Chemistry and Technology 39 (1966) 287-305.

[8] D.F. Moore, The Friction and Lubrication of Elastomers, Oxford, G.B., 1972

[9] C.T.R. Pulford, Failure of rubber by abrasion, Rubber Chemistry and Technology 58 (1984) 653-661.

[10] J.F. Archard, Contact and rubbing of flat surfaces, Journal of Applied Physics 24 (1953) 981-988.

[11] K.A. Grosch, A. Schallamach, The load dependence of laboratory abrasion and tyre wear, Kautschuk und Gummi Kunststoffe 22 (1969) 288-292.

[12] A. Schallamach, On the abrasion of rubber, Proceedings Institution of the Physical Society - Section B 67B (1954) 883-891.

[13] G. Heinrich, Hysteresis friction of sliding rubbers on rough and fractal surfaces, Rubber Chemistry and Technology 70 (1996) 1-14.

[14] B.N.J. Persson, E. Tosatti, Qualitative theory of rubber friction and wear, Journal of Chemical Physics 112 (2000) 2021-2029.

[15] A. Schallamach, Abrasion of rubber by a needle, Journal of Polymer Science 9 (1952) 385-404.

[16] A.G. Thomas, Factors influencing the strength of rubbers, Rubber Chemistry and Technology 48 (1975) 902-912.

[17] A. Schallamach, Abrasion pattern on rubber, Transactions Institution of the Rubber Industry 28 (1952) 256-268.

[18] P. Thirion, R. Chasset, Le frottement du caoutchouc, Institut Français du caoutchouc (I.F.C.), 1964

[19] A.K. Bhowmick, Ridge formation during the abrasion of elastomers, Rubber Chemistry and Technology 55 (1982) 1055-1062.

[20] Y.S. Kim, H.S. Byun, S. Kim, A.N. Gent, Abrasion of selected rubber compounds with a DIN abrader, Korea Polymer Journal 7 (1999) 116-123.

[21] R. Ramakrishnan, J.A. Donovan, The effect of abrading surfaces on the wear of rubber tread compounds, Rubber Chemistry and Technology 68 (1995) 609-622.

[22] T. Iwai, Y. Uchiyama, K. Shimosaka, K. Takase, Study on the formation of periodic ridges on the rubber surface by friction and wear monitoring, Wear 259 (2005) 669-675.

[23] M.M. Reznikovskii, G.I. Brodskii, Feature of the mechanism of abrasion of highly elastic materials, in: D.I. James (Ed.), Abrasion of Rubber, McLaren Palmerton, 1967.

[24] Y. Uchiyama, Wear of rubber through roll formation, International Polymer Science and Technology 12 (1985) 51-62.

[25] Y. Fukahori, H. Yamazaki, Mechanism of rubber abrasion. 1. Abrasion patternformation in natural-rubber vulcanizate, Wear 171 (1994) 195-202.

[26] K.L. Johnson, Contact Mechanics, Cambridge University Press, 1985.

[27] A.I. Medalia, Effect of carbon black on ultimate properties of rubber vulcanizates, Rubber Chemistry and Technology 60 (1986) 45-61.

[28] L.E. Nielsen, R.F. Landel, Mechanical Properties of Polymers and Composites, Marcel Dekker, New York, 1994.

[29] M.J. Wang, Effect of polymer-filler and filler-filler interactions on dynamic properties of filled vulcanizates, Rubber Chemistry and Technology 71 (1998) $520-589$.

[30] G. Heinrich, M. Klüppel, Reinforcement of elastomers, Advances in Polymer Science 160 (2002) 1-44.

[31] A.R. Payne, Journal of Applied Polymer Science 7 (1963) 873-885.

[32] J.A.C. Harwood, L. Mullins, A.R. Payne, Journal of Applied Polymer Science 9 (1965) 3011-3021.

[33] G. Kraus, Reinforcement of Elastomers, Interscience Publishers, New York, 1965. 
[34] J.D. Ferry, Viscoelastic Properties of Polymers, Wiley, New York, 1980.

[35] J.G. Curro, P. Pincus, Macromolecules 16 (1983) 559-562.

[36] A.I. Medalia, Rubber World 168 (1973) 49.

[37] Y. Bomal, S. Touzet, R. Barruel, P. Cochet, B. Dejean, Developments in silica usage for decreased tyre rolling resistance, Kautschuk Gummi Kunststoffe 50 (1997) 434.

[38] J. Berriot, H. Montès, F. Lequeux, D.R. Long, P. Sotta, L. Monnerie, Gradient of glass transition temperature in filled elastomers, Europhysics Letters 64 (2003) 50-56.

[39] S. Merabia, P. Sotta, D.R. Long, A microscopic model for the reinforcement and the nonlinear behavior of filled elastomers and thermoplastic elastomers (Payne and Mullins Effects), Macromolecules 41 (2008) 8252-8266.

[40] J. López-Valentín, I. Mora-Barrantes, J. Carretero-González, M.A. LópezManchado, P. Sotta, D.R. Long, K. Saalwächter, Novel experimental approach to evaluate filler-elastomer interactions, Macromolecules 43 (2010) 334-346.

[41] A. Vieyres, R. Pérez-Aparicio, P.-A. Albouy, O. Sanseau, K. Saalwächter D.R. Long, P. Sotta, Sulfur-cured natural rubber elastomer networks: correlating crosslink density, chain orientation and mechanical response by combined techniques, Macromolecules 46 (2013) 889-899.
[42] G. Kraus, Mechanical losses in carbon black filled elastomers, Journal of Applied Polymer Science 39 (1984) 75-92.

[43] E. Barthel, A. Perriot, A. Chateauminois, C. Frétigny, Elastic contact to nearly incompressible coatings: stiffness enhancement and elastic pile-up, Philosophical Magazine 33-35 (2006) 5359-5369.

[44] E. Gacoin, C. Frétigny, A. Chateauminois, A. Perriot, E. Barthel, Measurement of the mechanical properties of thin films mechanically confined within contacts, Tribology Letters 21 (2006) 245-252.

[45] C. Frétigny, A. Chateauminois, Solution for the elastic field in a layered medium under axisymmetric contact loading, Journal of Physics D: Applied Physics 40 (2007) 5418-5426.

[46] F. Yang, Axisymmetric indentation of an incompressible elastic thin film, Journal of Physics D: Applied Physics 36 (2003) 50-55.

[47] H. Liang, Y. Fukahori, A.G. Thomas, J.J.C. Busfield, Rubber abrasion at steady state, Wear 266 (2009) 288-296.

[48] H. Liang, Y. Fukahori, A.G. Thomas, J.J.C. Busfield, The steady state abrasion of rubber: why are the weakest rubber compounds so good in abrasion? Wear 268 (2010) 756-762. 